\title{
Single-layer crystalline phases of antimony: Antimonenes
}

\author{
O. Üzengi Aktürk, ${ }^{1}$ V. Ongun Özçelik, ${ }^{2,3}$ and S. Ciraci ${ }^{4, *}$ \\ ${ }^{1}$ Department of Physics, Adnan Menderes University, 09100 Aydın, Turkey \\ ${ }^{2}$ UNAM-National Nanotechnology Research Center, Bilkent University, 06800 Ankara, Turkey \\ ${ }^{3}$ Institute of Materials Science and Nanotechnology, Bilkent University, 06800 Ankara, Turkey \\ ${ }^{4}$ Department of Physics, Bilkent University, 06800 Ankara, Turkey \\ (Received 24 April 2015; revised manuscript received 6 June 2015; published 25 June 2015)
}

\begin{abstract}
The pseudolayered character of 3D bulk crystals of antimony has led us to predict its 2D single-layer crystalline phase named antimonene in a buckled honeycomb structure like silicene. Sb atoms also form an asymmetric washboard structure like black phospherene. Based on an extensive analysis comprising $a b$ initio phonon and finite-temperature molecular dynamics calculations, we show that these two single-layer phases are robust and can remain stable at high temperatures. They are nonmagnetic semiconductors with band gaps ranging from $0.3 \mathrm{eV}$ to $1.5 \mathrm{eV}$, and are suitable for $2 \mathrm{D}$ electronic applications. The washboard antimonene displays strongly directional mechanical properties, which may give rise to a strong influence of strain on the electronic properties. Single-layer antimonene phases form bilayer and trilayer structures with wide interlayer spacings. In multilayers, this spacing is reduced and eventually the structure changes to 3D pseudolayered bulk crystals. The zigzag and armchair nanoribbons of the antimonene phases have fundamental band gaps derived from reconstructed edge states and display a diversity of magnetic and electronic properties depending on their width and edge geometry. Their band gaps are tunable with the widths of the nanoribbons. When grown on substrates, such as germanene or $\mathrm{Ge}(111)$, the buckled antimonene attains a significant influence of substrates.
\end{abstract}

DOI: 10.1103/PhysRevB.91.235446

PACS number(s): 31.15.A-, 81.05.Zx, 68.65.-k, 81.05.ue

\section{INTRODUCTION}

The search for the contender of graphene has led to the prediction/synthesis of new single-layer, crystalline nanostructures and their van der Waals heterostructures, which do not exist in nature. Silicene, germanene, and stanene, namely graphene analogs of group IV elements [1-5]; h-BN analogs of group IV-IV, III-V, and II-VI compounds [6]; graphyne [7-9]; and the auxetic piezoelectric 2D material silicatene with negative Poisson's ratio [10,11], have been actively studied. Additionally, transition-metal dichalcogenides have proven to be superior to graphene in specific optoelectronic applications [12-15]. More recently, the fabrication of field effect transistors using micrometer-sized flakes consisting of two to three layers of black phosphorus [16] and theoretical analysis $[17,18]$ revealing the stability of its single-layer allotropes brought group V elements into the focus.

Our theoretical study exploiting the idea of whether other group $\mathrm{V}$ elements can form single-layer structures has revealed that $\mathrm{Sb}, \mathrm{As}$, and $\mathrm{N}$ atoms can indeed form $2 \mathrm{D}$ crystalline structures like silicene or black phosphorene. This paper reports that 2D, single-layer crystalline phases of antimony, named antimonene, can remain stable. Moreover, their 1D ribbons, bilayers, trilayers, and multilayers are also stable and display electronic and magnetic properties which may lead to potential applications in emerging 2D electronics.

Here we first examined the 3D bulk crystal of Sb having the trigonal and hexagonal lattice with the $R 3-m h$ space group, which display a pseudolayered atomic configuration as if it were constructed by the stacking of the layers having buckled honeycomb structure. Using structure optimizations based on the conjugate gradient method we then revealed two single-layer phases of $\mathrm{Sb}$, namely buckled antimonene

*ciraci@fen.bilkent.edu.tr
(B-antimonene or simply B-Sb) similar to silicene and washboard antimonene (W-antimonene or simply W-Sb) similar to black phospherene. While $\mathrm{W}-\mathrm{Sb}$ is prone to longwavelength instability, an asymmetric washboard structure (aW-antimonene or simply aW-Sb) is found to have slightly lower energy. We carried out an extensive analysis to ensure that $\mathrm{B}-\mathrm{Sb}$ and aW-Sb single-layer structures are stable above room temperature. We examined the character of their bonding and investigated the effects of vacancy defects. We then characterized these phases, their quasi-1D nanoribbons, bilayers, and multilayers by calculating their mechanical, electronic, and magnetic properties. Furthermore we showed how these properties deduced for suspended antimonene are modified when antimonene was grown on substrates like germanene and $\mathrm{Ge}(111)$ surfaces. Our predictions are corroborated by the fabrication of thin antimony films [19-21].

\section{METHOD}

Our predictions regarding 3D bulk antimony and 2D single-layer antimonene phases, as well as their 1D and 3D structures, are obtained from first-principles, pseudopotential plane wave calculations based on the spin-polarized density functional theory (DFT). We used the generalized gradient approximation including van der Waals (vdW) corrections at the DFT-D2 level [22], which was found to be suitable for layered structures in our earlier tests [23]. Owing to the location of the $\mathrm{Sb}$ atom in the fifth row of the periodic table, spin-orbit coupling (SOC) is also included in specific calculations. Similar first-principles DFT studies carried out in the past have been successful in predicting several new 2D structures $[2,6,10,15]$, which were subsequently synthesized/fabricated. We used projector-augmented wave (PAW) potentials [24] and the exchange-correlation was approximated by the Perdew-Burke-Ernzerhof (PBE) functional [25]. While optimizing atomic positions using the conjugate gradient 
(CG) method, the energy convergence value between two consecutive steps was chosen as $10^{-5} \mathrm{eV}$ and the maximum Hellmann-Feynman force acting on each atom was less than $0.02 \mathrm{eV} / \AA$. Calculations were carried out using the VASP software [26]. A plane-wave basis set with kinetic energy cutoff of $300 \mathrm{eV}$ was used. The Brillouin zone (BZ) corresponding to the primitive unit cell was sampled by a fine grid [27] of $(11 \times 11 \times 9),(21 \times 21 \times 1)$, and $(21 \times 1 \times 1) \mathbf{k}$ points for 3D, 2D, and 1D systems, respectively. Both kinetic energy cutoff and $\mathbf{k}$-point sampling values are determined by performing extensive convergence analyses. For example, increasing their values used in the calculations by $20 \%$ resulted in changes in the energies by less than $10^{-5} \mathrm{eV}$.

Since the band gaps are underestimated by standard DFT, we also carried out calculations using the screened hybrid functional, HSE06 method [28]. In addition to ab initio phonon calculations [29], the stability of the optimized structures was tested at high temperatures by performing $a b$ initio, finite-temperature molecular dynamics (MD) calculations. A
Nosé thermostat is used and Newton's equation of motion is integrated through the Verlet algorithm with time steps of $2 \mathrm{fs}$.

\section{PSEUDOLAYERED 3D CRYSTAL OF Sb}

Antimony has a 3D crystal with trigonal and hexagonal lattice and $R 3-m h$ space group. Experimental values of this $3 \mathrm{D}$ crystalline structure are the cohesive energy, $E_{C}=$ $2.75 \mathrm{eV} /$ atom, and the lattice constants [30,31] at $298 \mathrm{~K}, a=$ $b=4.31 \AA, c=11.27 \AA$, and Sb-Sb bond distance $2.91 \AA$. Our calculated values obtained from full structure optimization are $E_{C}=3.12 \mathrm{eV} /$ atom, $a=b=4.28 \AA$, and $c=11.18 \AA$. In Fig. 1(a), the atomic configuration, together with the relevant structural parameters, is illustrated. This 3D bulk phase can be viewed as consisting of an ABCABC type of stacking of the single-layer structure. Each isolated single layer can be identified as a buckled honeycomb structure with threefold rotation axis passing through the center of the hexagons. Because of the ABC stacking, the Sb-Sb bonds exhibit a (a)

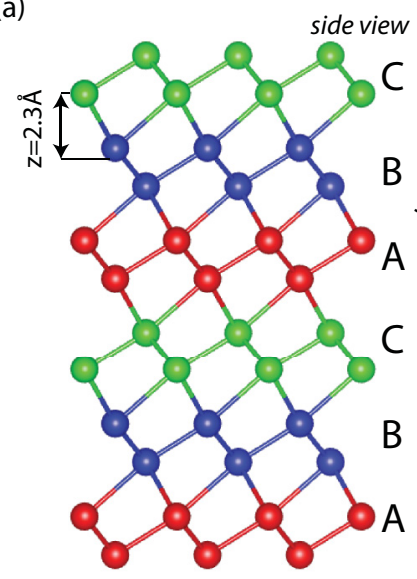

(c)

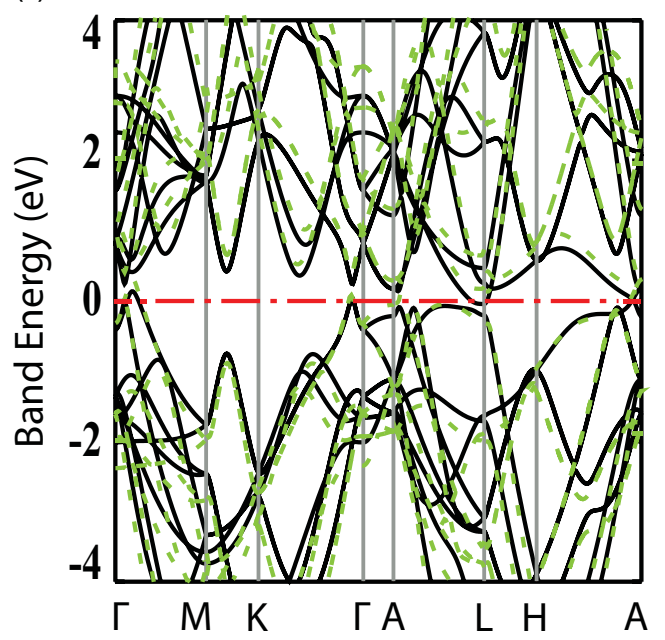

(b)

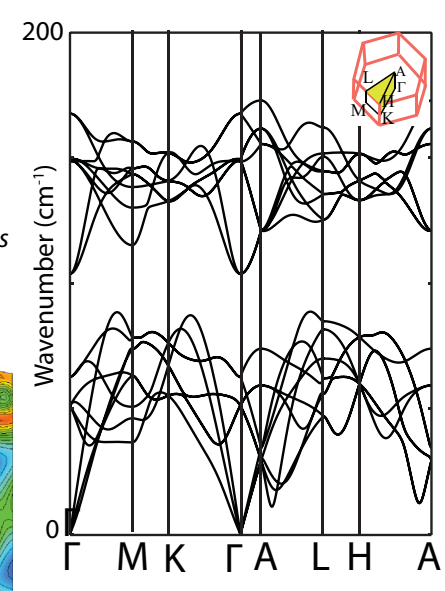

(d)

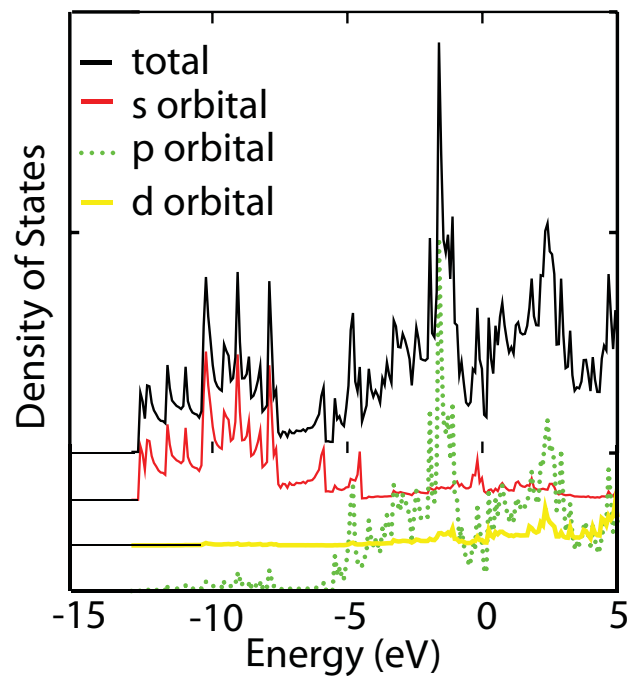

FIG. 1. (Color online) Results of ab initio calculations on the 3D bulk crystal of Sb with trigonal and hexagonal lattice. (a) Side and top views of the optimized atomic configuration and structural parameters. Pseudolayered character of the crystal is highlighted by atomic layers exhibiting $\mathrm{ABCABC}$... stacking. Inlayer and interlayer bonding is depicted by isosurface and contour plots of the total charge density. (b) Calculated dispersion relations of bands of vibrational frequencies. The Brillouin zone is shown by inset. (c) Electronic energy bands calculated within PBE. The correction by HSE is shown by green-dashed lines. The zero of energy is set to the Fermi level. (d) Total and orbital projected densities of states. For illustrative purposes, the zero of the vertical axis is shifted for each projected density of states. 
staggered arrangement in the top view. The smallest interlayer spacing between single layers is $z=2.3 \AA$ and hence it allows not only vdW attraction, but also significant chemical interaction. This is clarified by the contour plots of the total charge density on a plane including two atoms in different layers. In this respect, 3D Sb is different from graphite and $\mathrm{MoS}_{2}$, where interlayer binding is mainly vdW attraction. Because of significant interaction between layers we identify this $3 \mathrm{D}$ crystal as pseudolayered. The calculated bands of the vibration frequencies are presented in Fig. 1(b). As shown in Fig. 1(c), the highest valence band slightly raises above the Fermi level attributing a metallic (semimetallic) character to the $3 \mathrm{D}$ crystal of antimony. Corrections to DFT band energies using the HSE06 method confirmed this situation. Metallic (semimetallic) character is attained by coupling between adjacent layers of buckled honeycomb structure. The total and orbital projected densities of states (DOS) in Fig. 1(d) show that $5 p$-like orbital states dominate the top of the valence band and the bottom of the conduction band, whereas the lower part of the valence band is derived from $5 s$ orbitals. The pseudolayered character of the 3D Sb crystal has led us to consider first the 2D single-layer, buckled honeycomb structure of $\mathrm{Sb}$ similar to silicene [1,2].

\section{MONOLAYER B-, W-, AND aW-ANTIMONENE}

Considering the atomic structure of the layers in 3D bulk crystal in Fig. 1(a), we performed structure optimizations including lattice constants using the CG method, and found that the suspended buckled honeycomb structure with $2 \mathrm{D}$ hexagonal lattice can be indeed a candidate for a stable structure corresponding to a local minimum on the Born-Oppenheimer surface. We specify this single-layer structure as B-antimonene. To increase the degrees of freedom of structure optimization using $(1 \times 1)$ primitive cells, we repeated the structure optimization calculations using $(4 \times 4)$ supercells, which also ended with the same geometry and cohesive energy. Incidentally, the planar honeycomb structure did not survive after structure optimization despite its cohesive energy of $2.20 \mathrm{eV} /$ atom.

B-antimonene structure has a cohesive energy of $2.87 \mathrm{eV} /$ atom and formation energy of $-0.25 \mathrm{eV} /$ atom relative to the $3 \mathrm{D}$ pseudolayered $\mathrm{Sb}$ crystal structure (having $R 3-m h$ space group). In Fig. 2(a), the atomic structure, 2D hexagonal lattice, primitive unit cell and various relevant structural parameters including bond angles $\alpha$ and $\beta$ are presented. In the B-antimonene structure, the $s p^{2}$ bonds of unstable planar honeycomb structure are rehybridized to maintain the stability through the buckling of $\Delta=1.67 \AA$, whereby the three $s p^{n \alpha}$ orbitals of one $\mathrm{Sb}$ atom having the bond angle $\alpha=89^{\circ}$ with three nearest $\mathrm{Sb}$ atoms form covalent $\sigma$ bonds and provide the rigidity of antimonene. The remaining two electrons on each $\mathrm{Sb}$ atom form $s p^{n \beta}$ orbitals with relatively high $p$ contribution, which are directed perpendicularly. They are involved in the $\pi$ - and $\pi^{*}$-like bonds between the pairs of up and down $\mathrm{Sb}$ atoms in the primitive unit cell. In this respect, unlike graphene and silicene, which have unsaturated metallic bonds, the bonds of B-antimonene are saturated. The bonding of B-antimonene is illustrated by the isosurfaces of the total charge density in Fig. 2(a).

The mechanical properties of B-antimonene are characterized by its in-plane stiffness, $C=A_{0}^{-1} \delta^{2} E_{T} / \delta \epsilon_{T}^{2}$, and Poisson's ratio, $v=-\epsilon_{x} / \epsilon_{y}, A_{0}$ being the equilibrium area of the unit cell. The calculated values are $C_{x}=C_{y}=41 \mathrm{~N} / \mathrm{m}$ and $v_{x y}=v_{y x}=0.21$. Apparently, the stiffness of $\mathrm{B}-\mathrm{Sb}$ is much lower than that of graphene (with $C=350 \mathrm{~N} / \mathrm{m}$ ) or BN (with $C=240 \mathrm{~N} / \mathrm{m}$ ), but comparable to that of silicene (with $C=$ $65 \mathrm{~N} / \mathrm{m}^{2}$ ). Antimony being a fifth-row element makes longer but weaker bonds as compared to the second-row elements.

The optimized atomic structure with relevant structural parameters and the bonding of the single-layer, symmetric washboard structure, namely $\mathrm{W}$-antimonene, are described (a) B-antimonene

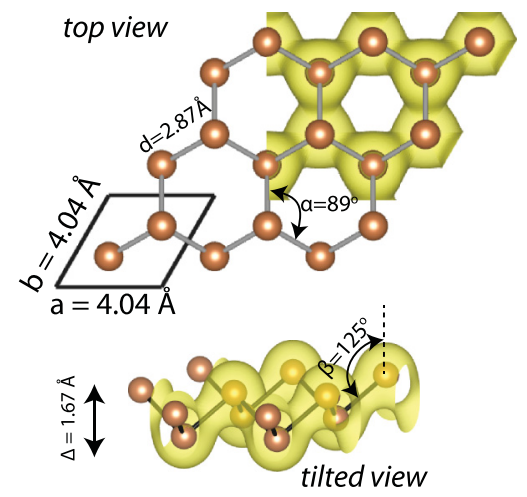

(b) W-antimonene

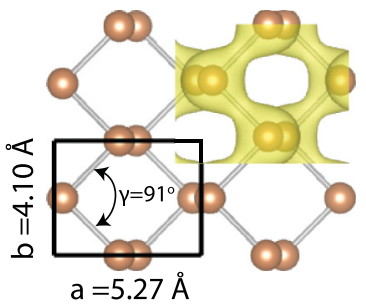

top view

c) aW-antimonene

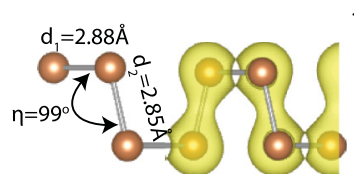

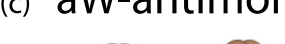

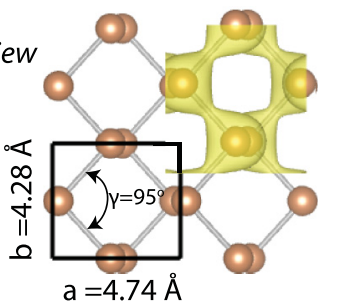

side view

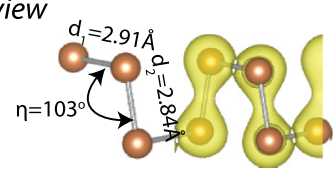

FIG. 2. (Color online) 2D, single-layer structures of antimony. (a) The equilibrium 2D crystalline structure of buckled honeycomb structure, i.e., B-antimonene, with hexagonal lattice. The primitive unit cell has two Sb atoms. Optimized values of the structural parameters, such as lattice constants, bond lengths, and bond angles, are also shown. Bonding between Sb atoms is depicted by the isosurfaces and contour plots of the total charge density. $\Delta$ is the buckling, where $\mathrm{Sb}$ atoms on the corners of the hexagon alternatively move up and down. (b) Same for 2D, symmetric washboard structure, i.e., W-antimonene, having 2D rectangular lattice. Rectangular primitive unit cell has four $\mathrm{Sb}$ atoms. In the side view one deduces two atomic planes. (c) Same for 2D, asymmetric washboard structure, i.e., aW-antimonene, with rectangular lattice. The primitive unit cell has four $\mathrm{Sb}$ atoms; single-layer structure is composed of four atomic planes. 
TABLE I. Values of relevant structural parameters, in-plane stiffness components, and energy band gaps calculated for B-Sb, W-Sb, aW-Sb phases of antimonene. Lattice constants, $a$ and $b$; nearest-neighbor distances, $d_{1}$ and $d_{2}$; buckling parameter, $\Delta$; angle between neighboring bonds, $\alpha, \beta, \gamma$, and $\eta$; components of in-plane stiffness, $C$; energy band gap, $E_{g}$, at PBE and HSE level. For the description of the structural parameters see Fig. 2.

\begin{tabular}{lcccccccccc}
\hline \hline Structure & $a, b(\AA)$ & $d_{1}(\AA)$ & $d_{2}(\AA)$ & $\Delta(\AA)$ & $\alpha(\mathrm{deg})$ & $\beta(\mathrm{deg})$ & $\gamma(\mathrm{deg})$ & $\eta(\mathrm{deg})$ & $C(\mathrm{~N} / \mathrm{m})$ & $E_{g-\mathrm{PBE}} / E_{g-\mathrm{HSE}}(\mathrm{eV})$ \\
\hline $\mathrm{B}-\mathrm{Sb}$ & $a=4.04, b=4.04$ & 2.87 & 2.87 & 1.67 & 89 & 125 & & & $C_{x}=41, C_{y}=41$ \\
$\mathrm{~W}-\mathrm{Sb}$ & $a=5.27, b=4.10$ & 2.88 & 2.85 & & & & 91 & 99 & $C_{x}=14, C_{y}=34$ & $0.37 / 0.89$ \\
$\mathrm{aW}-\mathrm{Sb}$ & $a=4.74, b=4.28$ & 2.91 & 2.84 & & & & 95 & 103 & $C_{x}=12, C_{y}=29$ & $0.16 / 0.34$ \\
\hline \hline
\end{tabular}

in Fig. 2(b). This structure has a $2 \mathrm{D}$ rectangular lattice like black phospherene and contains four $\mathrm{Sb}$ atoms in the primitive unit cell. Its calculated cohesive energy and formation energy are $2.88 \mathrm{eV} /$ atom and $-0.24 \mathrm{eV} /$ atom, respectively. Like $\mathrm{B}$-antimone, each $\mathrm{Sb}$ atom is threefold coordinated with in-plane $\mathrm{Sb}-\mathrm{Sb}$ bond distance of $2.85 \AA$ and interplanar $\mathrm{Sb}-\mathrm{Sb}$ bond distance of $2.88 \AA$. The corresponding bond angles are $\gamma=91^{\circ}$ and $\eta=99^{\circ}$. Accordingly, the single-layer $\mathrm{W}$-antimonene consists of two parallel atomic planes. This atomic configuration attributes directional elastic properties to W-antimonene, namely $C_{x}=14 \mathrm{~N} / \mathrm{m}$ and $v_{x y}=0.42$, but $C_{y}=34 \mathrm{~N} / \mathrm{m}$ and $v_{y x}=1.01$.

The total energy of symmetric $\mathrm{W}$-antimonene can be further lowered by $10 \mathrm{meV} /$ atom, if one of two $\mathrm{Sb}$ atoms, which were located in either plane in the primitive unit cell of symmetric $\mathrm{W}-\mathrm{Sb}$, are slightly displaced outwards and also the lattice constants of the rectangular primitive cell are modified. This asymmetric reconstruction takes place to prevent the structure from the long-wavelength instability, as we discuss in the following sections. We specify the asymmetric structure as aW-antimonene in the rest of the paper. In Fig. 2(c) we describe the atomic structure of aW-Sb together with its relevant structural parameters and isosurfaces of total charge distribution. Similarly to $\mathrm{W}-\mathrm{Sb}$, aW-Sb has strongly directional mechanical properties with $C_{x}=12 \mathrm{~N} / \mathrm{m}$ and $v_{x y}=0.36$, but $C_{y}=$ $29 \mathrm{~N} / \mathrm{m}$ and $v_{y x}=1.20$. High Poisson's ratio is indigenous to the washboard structure. In Table I, relevant structural parameters, in-plane stiffness components, and band gaps calculated for three single-layer phases of antimonene are summarized.

\section{A. Stability analysis}

Rather high cohesive energies of optimized structures at $T=0 \mathrm{~K}$ may not ensure that $2 \mathrm{D}$ antimonene phases correspond to deep minima in the Born-Oppenheimer surface.
In fact, these 2D structures may undergo instabilities due to long-wavelength lateral/transversal displacements, as well as thermal excitations at high temperatures. The most critical issue we have to clarify is whether these $2 \mathrm{D}$ antimonene phases are stable and remain stable at high temperature. In order to verify that $\mathrm{B}-, \mathrm{W}$-, and aW-antimonene remain stable against low-frequency acoustical vibrations inducing long-wavelength transverse/longitudinal displacements in different directions of the BZ, we calculated the frequencies of vibrational modes using a fine-grained k-point sampling. The vibrational bands including the ZA band along symmetry directions of the BZ are presented in Figs. 3(a)-3(c). The optical branches are well separated from the acoustical branches. All calculated frequencies are positive ensuring the stability of B-antimonene. However, for the symmetric $\mathrm{W}-\mathrm{Sb}$, the vibrational frequencies of specific branches become imaginary as $\mathbf{k} \rightarrow 0$ as shown in Fig. 3(b). This indicates instability in the W-Sb structure for long-wavelength acoustical vibrations. This instability has been restored through the reconstruction of the atomic structure leading to aW-Sb; all of its modes attained positive vibrational frequencies in Fig. 3(c).

Even if the stability is assured through the positive frequencies of all vibrational modes in the BZ, the structure may correspond to a shallow local minimum on the Born-Oppenheimer surface. Thus, the structure can be prone to instability at finite temperature. Further to the calculations of vibrational frequencies, we performed ab initio MD calculations to test the stability at high temperature. First, both structures remained stable at $700 \mathrm{~K}$ for $3 \mathrm{ps}$. Then the temperature increased to $1000 \mathrm{~K}$. Both B-Sb and aW-Sb structures remained stable in $a b$ initio MD simulations lasting 2 ps at $1000 \mathrm{~K}$. These MD results indicate that both antimonene structures can maintain their stability at least above room temperature and hence the local minima corresponding to these phases are deep enough to sustain their various applications above room temperature.

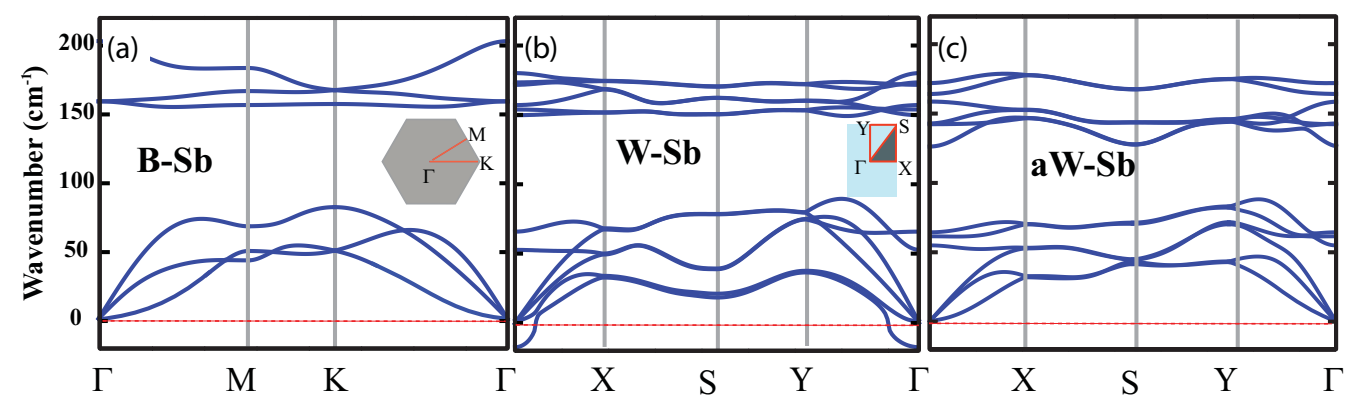

FIG. 3. (Color online) Ab initio calculations of the bands of vibrational frequencies. The Brillouin zones and their symmetry directions are shown by insets. (a) B-antimonene. (b) W-antimonene with imaginary frequencies as $\mathbf{k} \rightarrow 0$. (c) aW-antimonene. 
Next we address the question of whether the stability of antimonene phases is affected by vacancy defects. The isolated vacancy defects are treated by periodically repeating them in a $5 \times 5$ supercell of antimonene, whereby the coupling with nearest defects is minimized. For a single-vacancy defect in $\mathrm{B}-\mathrm{Sb}$, three twofold coordinated $\mathrm{Sb}$ atoms around the vacancy are relaxed and form an equilateral triangle, whereby the second-nearest-neighbor distance is reduced from $4.05 \AA$ to $3.72 \AA$. Accordingly, the structure around the vacancy is squeezed, but remains symmetric without rebonding of surrounding atoms. This situation is in contrast to the vacancy in graphene, where reconstruction or rebonding of carbon atoms surrounding the vacancy is observed [32-34]. The vacancy formation is an endothermic process and the formation energy of the single vacancy in B-Sb is $5.03 \mathrm{eV}$. At the end, the defect structure remains stable by gaining permanent magnetic moment $\mu=1.0 \mu_{B}$ per vacancy. The single-vacancy defect in aW-Sb displays similar behavior. Vacancy formation energy is relatively smaller and is $4.22 \mathrm{eV}$. Three $\mathrm{Sb}$ atoms surrounding the vacancy undergo a relaxation without rebonding. They form a triangle with edges of $\sim 3.60 \AA$. The single vacancy in the aW-Sb structure attain also a permanent magnetic moment of $0.6 \mu_{B}$ per vacancy. Clearly, like B-Sb, the single-vacancy defect does not cause any instability in aW-Sb.

\section{B. Electronic band structure}

We gain insight into electronic properties of antimonene phases. As shown in Fig. 4(a), B-antimonene is a nonmagnetic insulator with an indirect band gap of $1.04 \mathrm{eV}$ calculated within PBE approximation, which occurs between the minimum of the conduction band along the $\Gamma-M$ direction and maximum of the valance band at the $\Gamma$ point. Calculations carried out by HSE show that the DFT band gap is underestimated by $\sim 0.51 \mathrm{eV}$; hence this indirect band gap increases to $1.55 \mathrm{eV}$ after HSE correction. As shown by the inset in Fig. 4(a), under the spin-orbit coupling the degeneracies at the top of the valance band are removed to split the bands further and the indirect band gap is reduced by $0.25 \mathrm{eV}$. Apparently, the bad gap of $\mathrm{B}$-antimonene lies in the range, which is convenient for several electronic applications. In this respect B-antimonene keeps the promise of potential applications in 2D flexible electronics. The total and $s-, p$-, and $d$-orbital projected densities of states indicate that $5 p$ orbital states dominate the top of the valence and the bottom of the conduction bands. While the contribution $s$-orbital states increase in lower parts of the valence band, $d$-orbital states contribute to the upper parts of the conduction band.

We also discuss the calculated electronic structure of symmetric $\mathrm{W}$-antimonene for the sake of completeness. In Fig. 4(b), the indirect band gap occurs between the minimum of the conduction band at the $\Gamma$ point and the maximum of the valance band along the $\Gamma-X$ direction. The band gap as calculated within the PBE approximation is found to be $0.37 \mathrm{eV}$, which increases to $0.84 \mathrm{eV}$ after HSE correction. In Fig. 4(c), the band gap of the aW-antimonene decreases to $0.16 \mathrm{eV}$ upon asymmetric $\mathrm{Sb}-\mathrm{Sb}$ bond formation. After HSE correction this band gap increases to $0.34 \mathrm{eV}$. The direct band gap calculated by PBE increases from $0.16 \mathrm{eV}$ to $0.19 \mathrm{eV}$, when the spin-orbit coupling is taken into account.

\section{Antimonene on substrates}

Since suspended single layers cannot form by themselves, they can be grown on suitable substrates. Under these circumstances, the substrate-antimonene interaction becomes crucial (a) B-antimonene
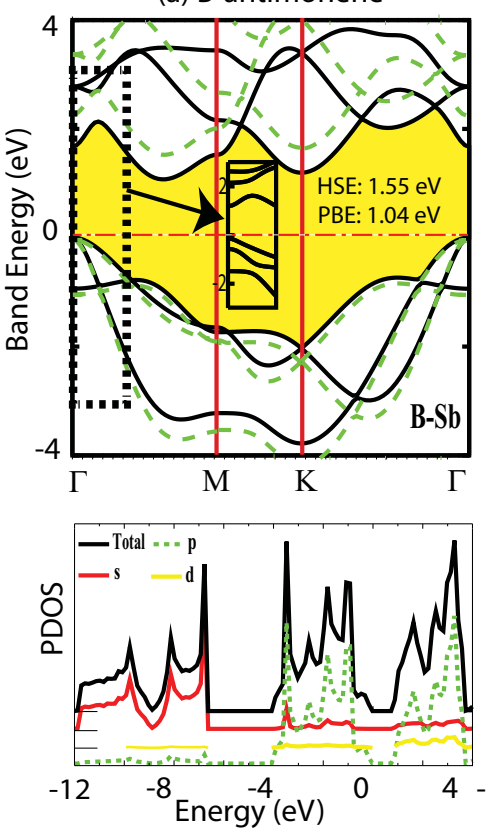

(b) W-antimonene
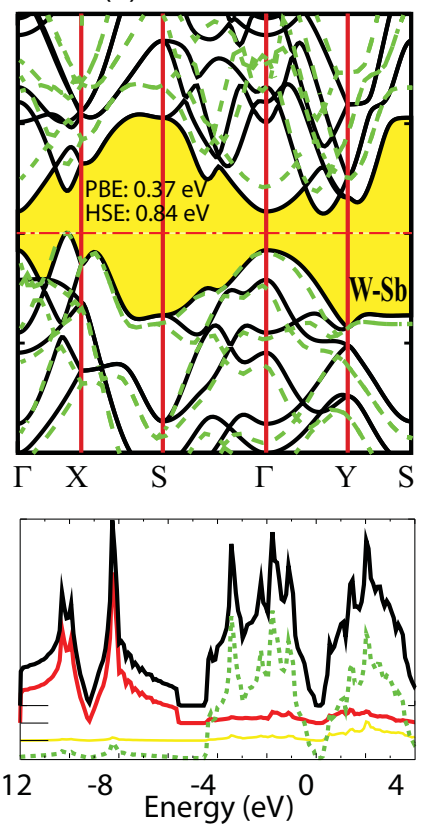

(c) aW-antimonene
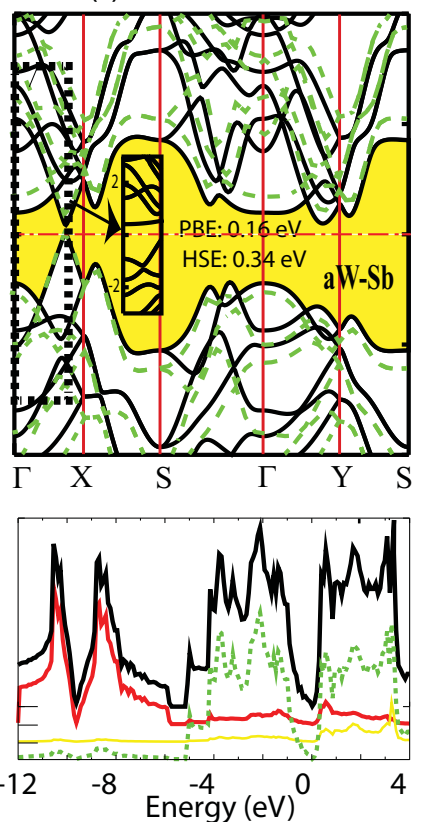

FIG. 4. (Color online) The electronic band structure together with the total and orbital projected densities of states of the single-layer antimonene phases. Zeros of the band energy are set at the maximum of the valance bands. Bands corrected by HSE are shown by green-dashed lines. Bands calculated by including spin-orbit coupling are shown by insets. (a) B-antimonene. (b) W-antimonene. (c) aW-antimonene. For illustrative purposes, the zero of the vertical axis is shifted for each projected density of states. 

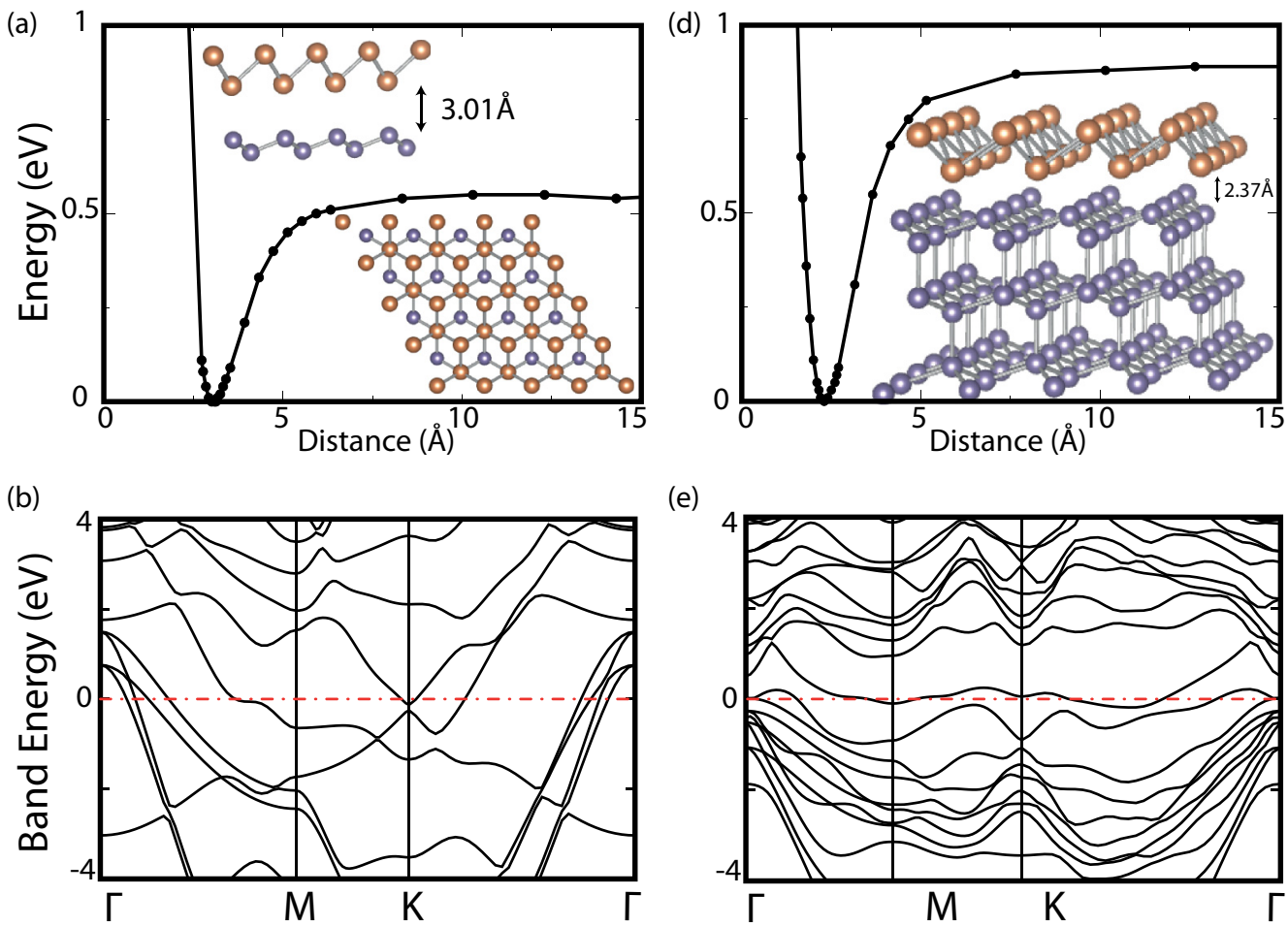

(e)

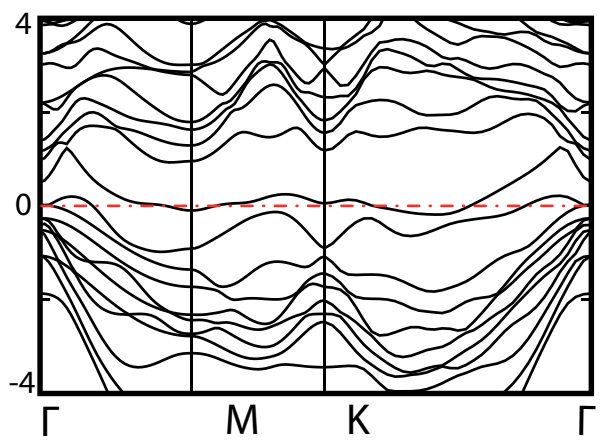

(c)

(f)
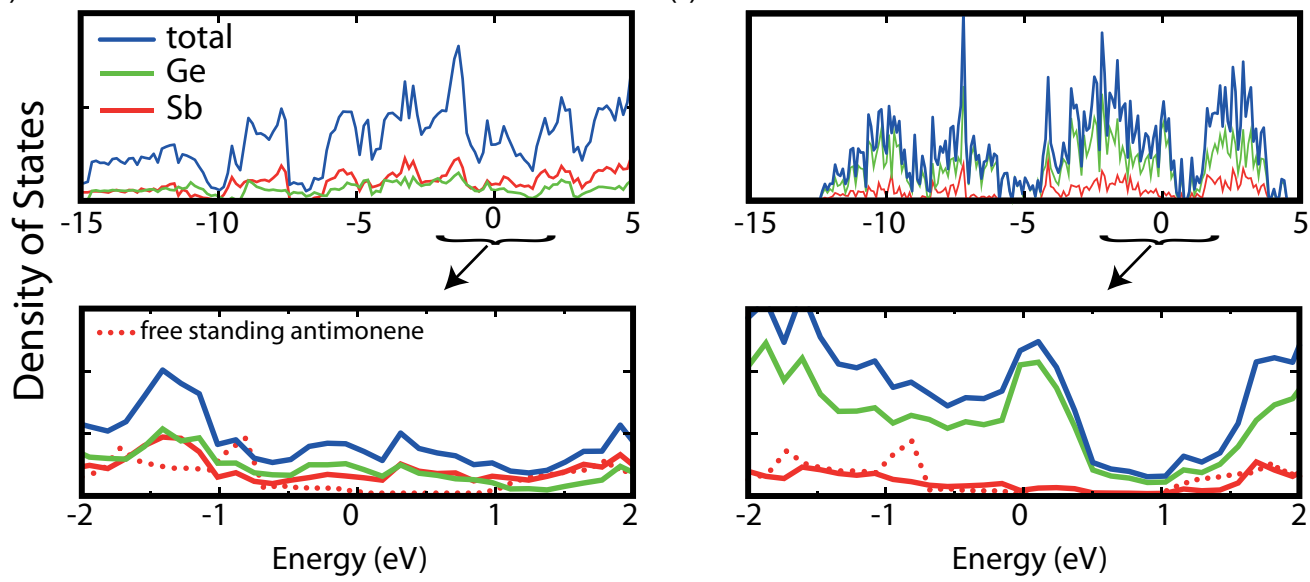

FIG. 5. (Color online) (a) The optimized atomic structure and binding interaction of B-antimonene grown on germanene surface (i.e., graphene-like structure of $\mathrm{Ge}$ atoms). The registry of $\mathrm{Sb}$ and $\mathrm{Ge}$ atoms are shown by inset. (b) Corresponding electronic energy band structure. (c) Total and partial densities of states (DOS) projected on $\mathrm{Sb}$ and $\mathrm{Ge}$ atoms. The comparison of the total density of states of free B-Sb single-layer structure with the density of states projected on the $\mathrm{Sb}$ atoms grown on germanene indicates significant substrate influence. (d)-(f) Same for B-antimonene grown on the Ge(111) substrate. The zero of energy is set to the Fermi level shown by dash-dotted line.

and may affect the properties revealed for the suspended single layers. To explore to what extent the properties of grown layers are influenced we investigated the binding energy and electronic band structure of $\mathrm{B}-\mathrm{Sb}$ grown on germanene and $\mathrm{Ge}(111)$ substrates, which are nearly lattice matched.

The atomic structure of $\mathrm{B}-\mathrm{Sb}$ grown on germanene with AA and $\mathrm{AB}$ stacking has been optimized. As shown in Fig. 5(a), we found that $\mathrm{AB}$ stacking is favorable energetically and has a binding energy of $250 \mathrm{meV}$ per $\mathrm{Sb}$ atom. The equilibrium separation between $\mathrm{Sb}$ and Ge layers is $z=3.01 \AA$. This material consisting of two single-layer honeycomb structures is a metal due to a significant interlayer coupling as shown in Fig. 5(b). The comparison made between total DOS of free $\mathrm{B}-\mathrm{Sb}$ and $\mathrm{DOS}$ projected to $\mathrm{B}-\mathrm{Sb}$ grown on germanene in Fig. 5(c) clearly indicates the influence of germanene substrate. The stability and significant interaction between germanene and antimonene suggests that composite materials derived from lateral stacking of $(\mathrm{B}-\mathrm{Sb})_{p} /(\text { germanene })_{q}(p$ and $q$ denote the numbers of layers) or their periodic alternation can be fabricated to achieve diverse functionalities. Notably, owing to significant interlayer chemical interaction this composite structure is rather different from van der Waals heterostructures. 
As an alternative substrate, the $\mathrm{Ge}(111)$ surface is considered, which is mimicked by a slab of bulk Ge consisting of three (111) atomic planes. The top and side views of the optimized atomic structure of $\mathrm{B}-\mathrm{Sb}$ on the $\mathrm{Ge}(111)$ substrate are shown by insets in Fig. 5(d), where the optimized total energy versus the spacing between $\mathrm{Ge}(111)$ and $\mathrm{B}-\mathrm{Sb}$ is also presented. The binding energy is $400 \mathrm{meV}$ per $\mathrm{Sb}$ atom, and the equilibrium separation is $2.37 \AA$. The energy bands corresponding to the equilibrium structure of B-antimonene grown on $\mathrm{Ge}(111)$ is metallic in Fig. 5(e). The total and projected densities of states and their comparison with the total density of states of the free B-Sb single layer in Fig. 5(f) clearly indicates significant substrate effects on the properties of antimonene. In particular, the high density of states of the grown $\mathrm{Sb}$ layer at the Fermi level is noted. While Ge and germanene engaged in significant interaction with the grown $\mathrm{B}-\mathrm{Sb}$ and they modified its electronic band structure, there might be substrates, which do not affect the properties of grown antimonene phases.

\section{BILAYER, TRILAYER, AND MULTILAYER ANTIMONENES}

For single-layer structures like $\mathrm{MoS}_{2}$ it is known that the electronic properties undergo gradual changes when additional layers are stacked above the first layer. To reveal whether similar changes can occur in antimonene, we investigated bilayer and trilayer structures of B-Sb. Furthermore, we explored whether the multilayer structure may be the precursor for the 3D graphitic structures or whether they can be associated with the thin slab of the 3D bulk crystal. The total energies of the bilayer and trilayer are calculated as a function of the interlayer distance $z$. For each value of $z$ the atomic positions and lattice constants are optimized. In Fig. 6(a) we show the variation of the total energy as a function of $z$. In the same figure is shown also the minimum-energy $\mathrm{AB}$ stacking of $\mathrm{B}$-antimonene single layers, whereby the $\mathrm{B}$ layer is displaced by $\mathbf{r}=(\mathbf{a}+\mathbf{b}) / 3$ relative to the adjacent A layer. The equilibrium spacing is found to be $z=3.81 \AA$, which is approximately $30 \%$ longer than the Sb-Sb bonds. The binding energy in equilibrium is $333 \mathrm{meV}$ per cell or $\sim 83 \mathrm{meV} /$ atom. This binding energy is also confirmed by calculating the difference of the total energies of the free $\mathrm{B}$-Sb single layer and of the bilayer from the expression, $E_{b}=E_{T}[B-\mathrm{Sb}] / 2-E_{T}[$ bilayer $] / 4$. The binding energy is composed of $\mathrm{vdW}$ and chemical interactions between two layers. However, the chemical interaction leads to significant changes in the electronic structure of the bilayer. Specifically, the semiconducting, single-layer B-antimonene changes into a metal when two of them are AB stacked to form a bilayer as shown in Fig. 6(b).

In the trilayer the interlayer interaction is relatively stronger and is calculated to be $0.7 \mathrm{eV}$ per cell or $\sim 117 \mathrm{meV} /$ atom, since the middle layer is capped from both sides. This is reflected to the equilibrium interlayer separation, which is calculated to be $z=3.65 \AA$. It is $0.16 \AA$ smaller than that of bilayer. The variation of the structure-optimized total energy as a function of $z$ is presented by the black (dark) curve in Fig. 6(c). The ABC stacking is found to be the most energetic stacking. Decreasing the interlayer separation of the trilayer $z$ from equilibrium value requires overcoming an energy barrier of $1.35 \mathrm{eV}$. Thereafter, a structural transformation takes place, where the interlayer separation is reduced suddenly to $z=2.5 \AA$ and the total energy drops to a second minimum. (a)

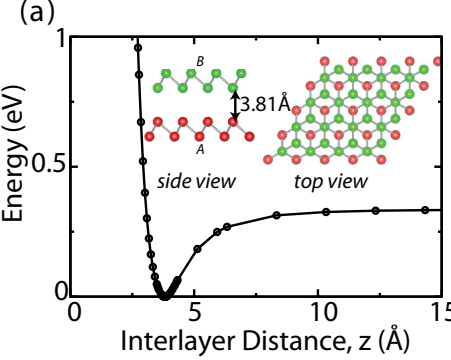

(b)

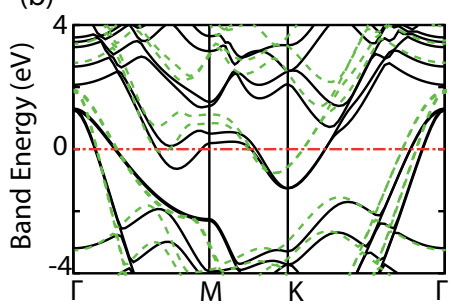

(c)

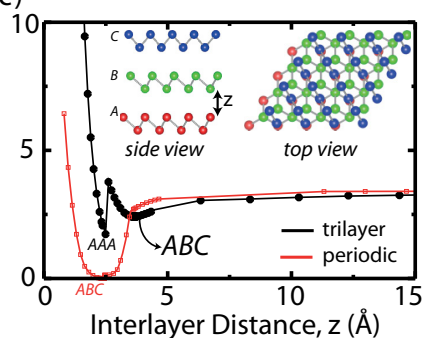

(d)

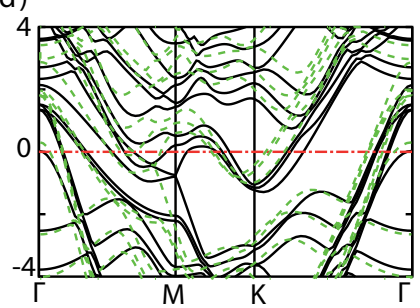

(e)

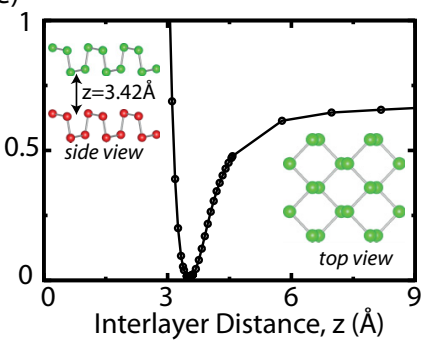

(f)

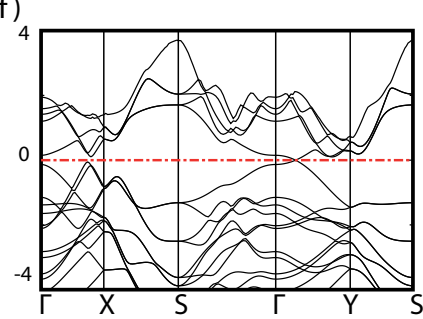

FIG. 6. (Color online) (a) Minimum-energy AB stacking geometry of the B-antimonene bilayer and the variation of its total energy with the distance $z$ between the layers. The total energies are given relative to $z \rightarrow$ infinity. (b) Energy band structure of bilayer corresponding to the equilibrium spacing. Calculations using HSE presented by green dashed lines. (c) Same for trilayer of B-antimonene in ABC stacking. The first minimum of the total energy occurs at $z=3.65 \AA$. Upon overcoming an energy barrier the second minimum occurs at $z=2.5 \AA$. The variation of the optimized total energy of the periodic 3D structure as a function of $z$, which exhibits a single minimum at $z=2.37 \AA$ corresponding to the pseudolayered 3D bulk crystal. (d) The energy band structure of the trilayer in the first minimum at $z=3.65 \AA$. (e) Minimum-energy AA stacking geometry of aW-antimonene bilayer and the variation of its total energy with the distance $z$ between layers. Top and side views of atomic structures are shown by insets. (f) Energy band structure of aW-antimonene bilayer in (e). 
In this transformation, the $\mathrm{ABC}$ stacking changes to either AAA or ABA stacking. The energy difference between these two different stacking geometries is minute, their binding energies approximately $113 \mathrm{meV} /$ atom stronger than that in the first minimum. Similar structural transformation is explored for multilayers, which are treated by a periodically repeating structure with ... $\mathrm{ABCABC} \ldots$ stacking as in the 3D pseudolayered bulk crystal in Sec. III. Here the structure-optimized total energy is calculated as a function of the interlayer spacing, $z$ ranging from $z=15 \AA$ to $z=2 \AA$. As shown with the red curve in Fig. 6(c), this periodic structure has a single minimum at $z=2.37 \AA$ with the binding energy of $3.40 \mathrm{eV}$ per cell; it is identical to the 3D pseudolayered structure. Accordingly, the single-layer B-antimonene does not construct a 3D graphitic structure with wide interlayer spacing; it rather replicates 3D pseudolayered bulk crystal as the number of antimonene layers increases. The electronic structure of the trilayer with $z=3.65 \AA$ is similar to that of bilayer and displays a metallic character in Fig. 6(d).

In the bilayer of aW-antimonene, the AA stacking attains the minimum energy. The equilibrium interlayer separation occurs at $z=3.42 \AA$ and the binding energy is $83 \mathrm{meV}$ as shown in Fig. 6(e). Similar to the bilayer of B-antimonene, the electronic structure exhibits a metallic character with bands crossing at the Fermi level along the $\Gamma-Y$ direction in Fig. 6(f).

Very recently, Zhang et al. [35] reported results of similar calculations on B-antimonene, and its bilayer and trilayer. They found the single layer $\mathrm{B}-\mathrm{Sb}$ as semiconductor. Their prediction for the fundamental band gap is $2.28 \mathrm{eV}$ with HSE correction. Also their energy bands for bilayer and trilayer have zero band gap. The present work, which has been carried out independently from the work by Zhang et al. [35], has a wider scope comprising not only B-Sb but also 3D bulk, W-Sb, and $\mathrm{aW}-\mathrm{Sb}$, and the nanoribbons of diverse edge geometries. Additionally, our study presents an extensive analysis of stability including finite-temperature $a b$ initio $\mathrm{MD}$ calculations and examines the interaction of B-antimonene grown on germanene and the $\mathrm{Ge}(111)$ surface, and treats antimonene nanoribbons as presented in forthcoming sections. In the present study the fundamental band gap was found to be $1.55 \mathrm{eV}$ with the HSE correction and $0.69 \mathrm{eV}$ with $\mathrm{PBE}+$ spin-orbit coupling.

\section{ELECTRONIC STRUCTURE OF ANTIMONENE NANORIBBONS}

Since finite-size ribbons or patches of antimonene will be used in various applications, their stability and electronic properties become relevant for the characterization of antimonene phases. Here we consider armchair and zigzag edge geometries, which are specified by the number of $\mathrm{Sb}$ atoms, $n$, in their primitive unit cell. In Fig. 7(a) we show the primitive unit cell and optimized atomic structure of the B-antimonene armchair nanoribbon with $n=22$ and the corresponding electronic energy band structure. Relaxations occur mainly at the edges. The B-antimonene nanoribbon has a nonmagnetic ground state. Two bands, each located at the edge of valence and conduction bands ( $\mathrm{V}$ and $\mathrm{C}$, respectively)

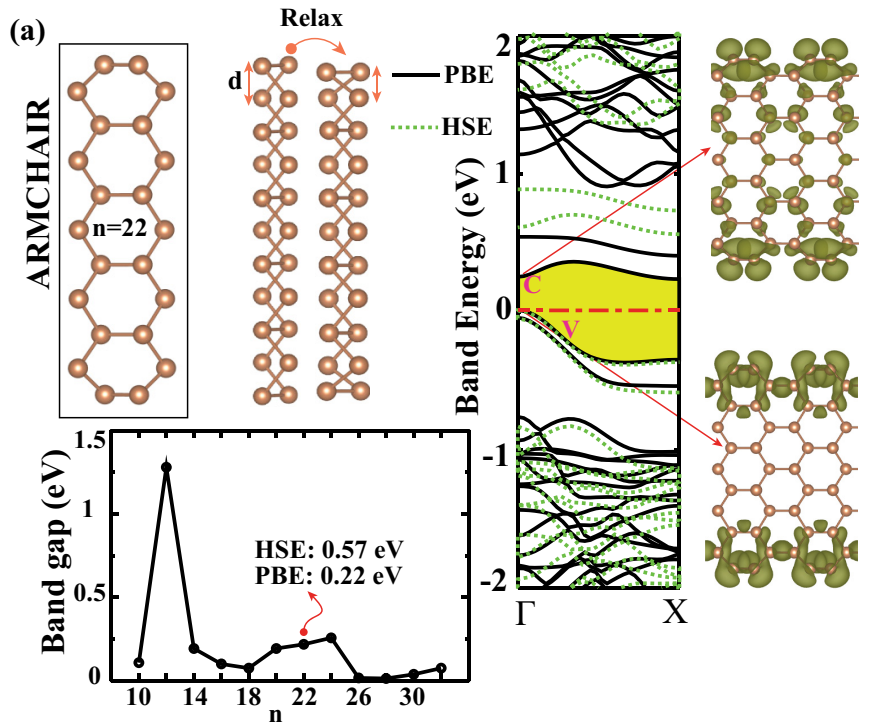

(b)

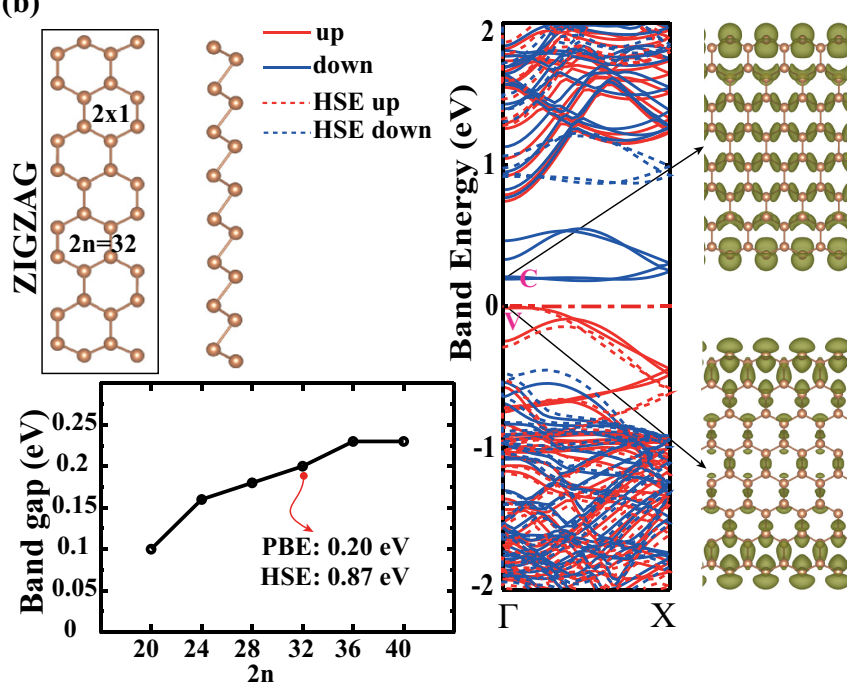

FIG. 7. (Color online) (a) The primitive unit cell, optimized atomic configuration, and energy band structure of the armchair $\mathrm{B}$-antimonene nanoribbon. The variation of band gap with $n$ and charge density isosurfaces of specific band states at the edges of conduction $(\mathrm{C})$ and valence $(\mathrm{V})$ bands are also shown. Energy bands corrected using HSE are shown by dashed lines. (b) Same for the zigzag B-antimonene nanoribbon. Structure optimization and band calculations are performed using $2 \times 1$ unit cell. The zero of energy is set at the top of the valence band. Spin-up and spin-down bands are shown by red and blue lines, respectively. Spin-up and spin-down bands corrected using HSE are also shown by dashed red and blue lines, respectively.

delineate the band gap in the momentum space. They are derived from the states localized at the edges as demonstrated by band-decomposed charge density isosurfaces. The PBE indirect band gap is calculated to be $E_{g}=0.218 \mathrm{eV}$, which increases to $E_{g}=0.574 \mathrm{eV}$ after the HSE correction. The variation of the PBE band gap $E_{g}$ with the width of the ribbon or $n$ is also shown.

Since the edge atoms of the zigzag B-Sb nanoribbon undergo a reconstruction, the structure optimization using 
the primitive unit cell with $n$ atoms mandates a fictitious, metallic structure. However, upon a Peierls-type structural transformation in the $2 \times 1$ cell, the metallic state changes to ferromagnetic semiconductor. Because of reconstruction the structure optimization and energy band structures are performed using the $2 \times 1$ unit cell. The atomic configuration, energy band structure, and charge density isosurfaces of specific states of the zigzag B-Sb with $2 n=32$ are presented in Fig. 7(b). The variation of the band gap with $2 n$ is also
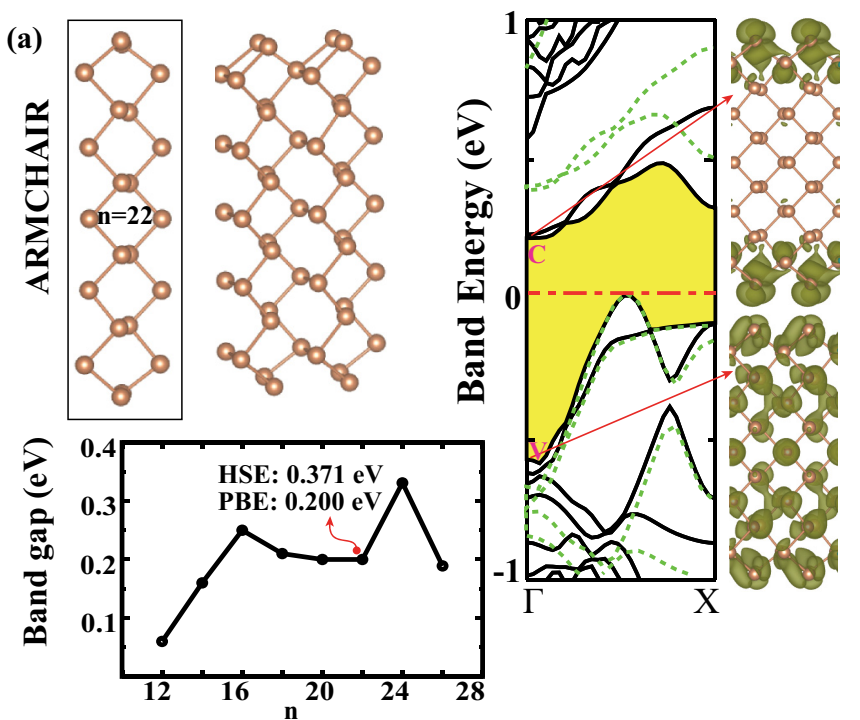

(b)

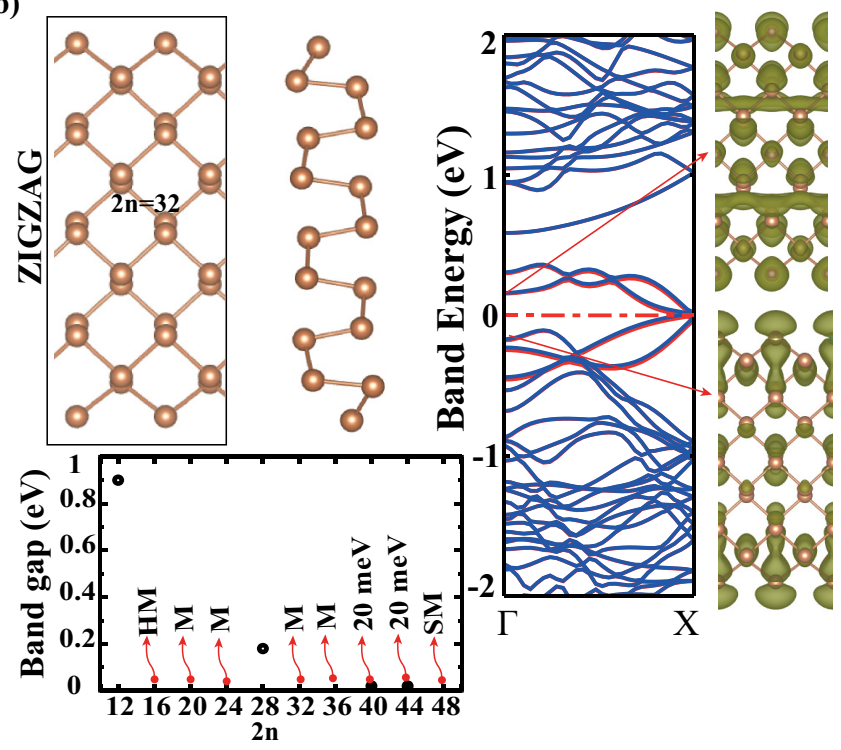

FIG. 8. (Color online) (a) The primitive unit cell having $n \mathrm{Sb}$ atoms, optimized atomic structure, and the energy band structure of the armchair aW-antimonene nanoribbon. The variation of band gap with $n$ and charge density isosurfaces of specific band states at the edges of conduction $(\mathrm{C})$ and valence $(\mathrm{V})$ bands are also shown. Energy bands calculated by HSE are shown by dashed lines. The zero of the energy is set at the top of the valence band. (b) Same for the zigzag aW-antimonene nanoribbon, where due to the reconstructions of edges calculations are performed using $2 \times 1$ unit cell. Spin-up and spin-down bands are shown by red and blue lines, respectively. The zero of energy is set at the Fermi level. given in the same figure. The small energy gap between filled spin-up and empty spin-down bands is calculated within PBE to be $E_{g}=0.200 \mathrm{eV}$. Upon HSE correction this band increases to $0.872 \mathrm{eV}$. Isosurfaces of charge density of specific states at the band edges demonstrate that they are constructed predominantly from the orbitals of edge atoms. Hence these states are localized at the edges of the nanoribbon. The PBE band gap is $\sim 0.1 \mathrm{eV}$ for $2 n=20$, but increases to $0.23 \mathrm{eV}$ for $2 n=40$.

In Fig. 8(a) we present the atomic configuration and electronic band structure of armchair and zigzag nanoribbons of aW-antimonene. The armchair nanoribbon with $n=22$ is a nonmagnetic semiconductor with a PBE band gap of $0.20 \mathrm{eV}$, which increases to $0.37 \mathrm{eV}$ after HSE correction. Isosurfaces of the selected states at the band edges illustrate the contribution of the edge atoms. The PBE bands vary between $0.1 \mathrm{eV}$ and $0.33 \mathrm{eV}$ depending on the values of $n$. The zigzag nanoribbon of aW-Sb is treated in a $2 \times 1$ unit cell due to edge reconstruction. For $2 n=32$ the zigzag nanoribbon is a metal. However, the character of the ribbon appears to strongly depend on its width. Depending on the values of $2 n$, the zigzag nanoribbon of aW-Sb can exhibit diverse properties, such as metallic, semimetallic, half-metallic, and semiconducting, as shown in in Fig. 8(b).

\section{CONCLUSIONS}

In conclusion, our theoretical study predicts that antimony atoms can form 2D single-layer crystal structures. One of these is named B-antimonene (similar to silicene). The singlelayer, symmetric washboard structure (similar to the black phosphorene) is, however, unstable against long-wavelength displacements. If the lattice constants of rectangular structure change and one of two $\mathrm{Sb}$ atoms on the top and bottom atomic planes of symmetric washboard structure are buckled, the total energy can be further lowered. The resulting new structure is called aW-antimonene. That these 2D single-layer B- and aW-antimonene structures are stable was concluded not only based on the structure optimizations achieved by the conjugate gradient method but also based on other tests, such as $a b$ initio calculation of vibration frequencies and $a b$ initio molecular dynamics calculations performed at temperatures as high as $1000 \mathrm{~K}$. We showed also that antimonene phases are robust against vacancy formation. B-antimonene and aW-antimonene are semiconductors with band gaps suitable for their use in 2D electronics. These single-layer phases of antimony can form stable bilayers, trilayers, and even multilayers. Because of significant interlayer coupling, bilayer and multilayer structures are metallic. We examined the effect of substrates for B-antimonene grown on germanene and the $\mathrm{Ge}(111)$ surface. We found a significant influence of these substrates on the grown B-antimonene, whereby the semiconducting single layer of antimonene changes into metal. Nanoribbons of antimonene phases display a diversity of properties depending on their width and edge geometry. Usually they are narrow band gap semiconductors and attain magnetic properties for zigzag edge geometry. Accordingly, the band gap can be engineered by varying the width of these nanoribbons. The stability and significant binding energy suggest the fabrication of composite materials 
consisting of vertically stacked antimonene and germanene heterostructures. Briefly, our study heralds stable structures derived from antimonene, such as multilayers and composites, 2D single layers, bilayers, trilayers, and 1D nanoribbons. They display diverse properties for various technological applications.

\section{ACKNOWLEDGMENTS}

The computational resources are provided by TUBITAK ULAKBIM, High Performance and Grid Computing Center (TR-Grid e-Infrastructure). V.O.Ö. and S.C. acknowledge financial support from the Academy of Sciences of Turkey (TUBA).
[1] E. Durgun, S. Tongay, and S. Ciraci, Phys. Rev. B 72, 075420 (2005).

[2] S. Cahangirov, M. Topsakal, E. Aktürk, H. Sahin, and S. Ciraci, Phys. Rev. Lett. 102, 236804 (2009).

[3] P. Vogt, P. DePadova, C. Quaresima, J. Avila, E. Frantzeskakis, M. C. Asensio, A. Resta, B. Ealet, and G. LeLay, Phys. Rev. Lett. 108, 155501 (2012).

[4] V. O. Özçelik, E. Durgun, and S. Ciraci, J. Phys. Chem. Lett. 5, 2694 (2014).

[5] Y. Xu, B. Yan, H. J. Zhang, J. Wang, G. Xu, P. Tang, W. Duan, and S. C. Zhang, Phys. Rev. Lett. 111, 136804 (2013).

[6] H. Sahin, S. Cahangirov, M. Topsakal, E. Bekaroglu, E. Akturk, R. T. Senger, and S. Ciraci, Phys. Rev. B 80, 155453 (2009).

[7] S. Tongay, S. Dag, E. Durgun, R. T. Senger, and S. Ciraci, J. Phys.: Condens. Matter 17, 3823 (2005).

[8] D. Malko, C. Neiss, F. Viñes, and A. Görling, Phys. Rev. Lett. 108, 086804 (2012).

[9] V. O. Özçelik and S. Ciraci, J. Phys. Chem. C 117, 2175 (2013).

[10] V. O. Özçelik, S. Cahangirov, and S. Ciraci, Phys. Rev. Lett. 112, 246803 (2014).

[11] X. Xu, J. Zhuang, Y. Du, H. Feng, N. Zhang, C. Liu, T. Lei, J. Wang, M. Spencer, T. Morishita, X. Wang, and S. X. Dou, Sci. Rep. 4, 7543 (2014).

[12] P. Joensen, R. F. Frindt, and S. R. Morrison, Mater. Res. Bull. 21, 457 (1986).

[13] J. N. Coleman, M. Lotya, A. O’Neill, S. D. Bergin, P. J. King, U. Khan, and K. Young, Science 331, 568 (2011).

[14] C. Ataca and S. Ciraci, J. Phys. Chem. C 115, 13303 (2011).

[15] C. Ataca, H. Sahin, and S. Ciraci, J. Phys. Chem. C 116, 8983 (2012).

[16] L. Li, Y. Yu, G. J. Ye, Q. Ge, X. Ou, H. Wu, D. Feng, X. H. Chen, and Y. Zhang, Nat. Nanotechnol. 9, 372 (2014).
[17] Z. Zhu and D. Tomanek, Phys. Rev. Lett. 112, 176802 (2014).

[18] T. Low, A. S. Rodin, A. Carvalho, Y. Jiang, H. Wang, F. Xia, and A. H. Castro Neto, Phys. Rev. B 90, 075434 (2014).

[19] C. Maanit, A. McKinley, and R. H. Williams, J. Phys. C: Solid State Phys. 18, 4975 (1985).

[20] M. W. Grant, P. F. Lyman, J. H. Hoogenraad, and L. E. Seiberling, Surf. Sci. 279, L180 (1992).

[21] R. Whittle, I. T. McGovern, D. R. T. Zahn, C. Müller, C. Nowak, A. Cafolla, and W. Braun, Appl. Surf. Sci. 56, 218 (1992).

[22] S. Grimme, J. Comput. Chem. 27, 1787 (2006).

[23] C. Ataca, M. Topsakal, E. Aktürk, and S. Ciraci, J. Phys. Chem. C 115, 16354 (2011).

[24] P. E. Blöchl, Phys. Rev. B 50, 17953 (1994).

[25] J. P. Perdew, K. Burke, and M. Ernzerhof, Phys. Rev. Lett. 77, 3865 (1996).

[26] G. Kresse and J. Furthmüller, Phys. Rev. B 54, 11169 (1996).

[27] H. J. Monkhorst and J. D. Pack, Phys. Rev. B 13, 5188 (1976).

[28] J. Paier, M. Marsman, K. Hummer, G. Kresse, I. C. Gerber, and J. G. Ángyán, J. Chem. Phys. 124, 154709 (2006).

[29] D. Alfe, Comput. Phys. Commun. 180, 2622 (2009).

[30] C. Kittel, Introduction to Solid State Physics, 8th ed. (John Wiley \& Sons, New York, 1996).

[31] C. S. Barrett, P. Cucka, and K. Haefner, Acta Cryst. 16, 451 (1963).

[32] O. V. Yazyev and L. Helm, Phys. Rev. B 75, 125408 (2007).

[33] A. W. Robertson, B. Montanari, K. He, C. S. Allen, Y. A. Wu, N. M. Harrison, A. I. Kirkland, and J. H. Warner, ACS Nano 7, 4495 (2013).

[34] V. O. Özçelik, H. H. Gurel, and S. Ciraci, Phys. Rev. B 88, 045440 (2013).

[35] Z. Zhang, Z. Yan, Y. Li, Z. Chen, and H. Zeng, Angew. Chem. Int. Ed. 54, 3112 (2015). 